\title{
Stochastic generation of homogeneous isotropic turbulence with well-defined spectra
}

\author{
A. Careta and F. Sagués \\ Departament de Química Física, Universitat de Barcelona, Diagonal 647, 08028 Barcelona, Spain \\ J. M. Sancho \\ Departament d'Estructura i Constituents de la Materia, Universitat de Barcelona, Diagonal 647, 08028 Barcelona, Spain
}

(Received 20 April 1993)

\begin{abstract}
A precise and simple computational model to generate well-behaved two-dimensional turbulent flows is presented. The whole approach rests on the use of stochastic differential equations and is general enough to reproduce a variety of energy spectra and spatiotemporal correlation functions. Analytical expressions for both the continuous and the discrete versions, together with simulation algorithms, are derived. Results for two relevant spectra, covering distinct ranges of wave numbers, are given.
\end{abstract}

PACS number(s): 02.70. $-\mathrm{c}$, 47.27.Eq, 05.40. $+\mathrm{j}$

\section{INTRODUCTION}

In many research areas of either fundamental or practical importance it is necessary to be able to compute or model turbulent velocity fields. Both the statistics of the turbulence and the particular turbulent structures of actual realizations of the flow field are commonly required.

In practice, most simulations of turbulent flow fields use exact or approximate solutions to the equations of motion and could be classified generically as "NavierStokes solvers" [1]. Although some progress has been made with the adaptation of spectral methods, at the present time direct numerical simulations of fully developed turbulence are restricted to low Reynolds numbers. For high Reynolds numbers, where the scale range exceeds that allowed by computer capacity, some scales must be discarded, and their influence upon the retained ones must be modeled. This is essentially the strategy of large-eddy simulations [2].

The main point we want to emphasize here is that, for many purposes, it suffices that the generated velocity fields retain only the most significative statistical features of fully developed turbulence: their spectra and correlation functions. Among the methods adapted to this perspective, we would like to briefly mention three of them. They are respectively based on random Fourier modes, random vortex models, and stochastic differential equations.

Simulations based on random Fourier modes were first introduced by Kraichnan in the early 1970s [3]. Kraichnan considered a Lagrangian description of the turbulent velocity field for both two-dimensional (2D) and 3D incompressible, stationary, homogeneous, and isotropic fluids. He treated the velocity field as a Gaussian variable modeled in terms of statistically independent random Fourier modes. In both cases he considered energy spectra describing widely distributed bands of excitations of Gaussian shape with a peak centered at a well-defined wave number. Additional modifications of this technique to consider the helicity of the fluid $[4,5]$ and to model the advection of small-scale eddies by the larger ones [6] have also been reported.

Similarly, random vortex models define the turbulent flow as the superposition of many statistically spacedistributed incompressible vortices. For instance, 2D frozen chaotic flows have been generated which allow the computation of $n$-point Eulerian correlation functions having well-defined spectra [7]. A time-dependent version of these models, where the velocity field satisfies symmetry requirements, has been reported in which the convection of vortices by the generated flow is taken into account [8].

Within the methods relying on stochastic differential equations, we could consider either the approach based on Navier-Stokes equations with additional noise terms [9] or the direct simulation of $3 \mathrm{D}$ velocity fields in the Lagrangian frame by means of an Ornstein-Uhlenbeck process [10]. In the latter approach, the random field appearing in the Langevin equation is generated in such a way that its spatial covariance matrix adopts the standard Eulerian form, but no attempt to reproduce a definite energy spectrum has been made.

Under the same perspective of using stochastic differential equations, we introduce here a versatile approach that can be applied to a variety of energy spectra and spatiotemporal correlation functions of well-behaved turbulent flows. We start with an auxiliary random scalar field $\eta(\mathbf{r}, t)$ whose temporal evolution is given by a generalization of the Ornstein-Uhlenbeck equation which incorporates spatial degrees of freedom. A trivial transformation is then employed to obtain a divergence-free, isotropic, stationary, and homogeneous stochastic $2 \mathrm{D}$ velocity field $\xi(\mathbf{r}, t)$ whose statistical parameters are easily identified in terms of the originally prescribed ones for $\eta(\mathbf{r}, t)$. A particular technique which largely facilitates the handling of the auxiliary field consists in simulating its dynamics in Fourier space [11]. This enables us to directly obtain the energy spectrum function, whereas transforming back to real-space generic expressions are derived for the spatiotemporal correlation function and its associated spatial and temporal correlation lengths. A simplified version of the procedure presented here has already been used in the context of the diffusion of passive 
scalars convected by turbulent flows [12].

Just to finish this introductory section, note that our approach, although here totally applied within the context of turbulence, is general enough to make possible a much wider range of application. Indeed the stochastic scalar field $\eta(\mathbf{r}, t)$ may be by itself a quantity of great interest, especially in those situations where one has to deal with spatiotemporal noise having finite correlations in both time and space. This would be the case in problems such as phase separation [13], interfacial growth [14], or fluctuational kinetics [15].

The paper is organized as follows. In Sec. II we introduce the model and the relevant statistical quantities in continuous space. Their discrete version is presented in Sec. III, whereas the appropriate simulation algorithms are derived in Sec. IV. Two examples of the general procedure that correspond, respectively, to the spectra originally proposed by Kraichnan [3] and by KármánObukhov [16,17], are illustrated in Sec. V. Final considerations are summarized in Sec. VI.

\section{THE CONTINUOUS MODEL}

As stated in the Introduction, we aim at obtaining a stochastic quantity which reproduces the most significative features of fully developed turbulence. Specifically, we are interested in the generation of zero mean, homogeneous, isotropic, and stationary 2D velocity fields $\xi(\mathbf{r}, t)$ characterized by well-behaved energy spectra $E(k)$. Accordingly, the quantity which will be of major importance for us is the two-point two-time correlation tensor $R^{i j}(r, s)$ defined by [18] (superscripts stand for components)

$$
R^{i j}(r, s)=\left\langle\xi^{i}\left(\mathbf{r}_{1}, t_{1}\right) \xi^{j}\left(\mathbf{r}_{2}, t_{2}\right)\right\rangle
$$

where $r=\left|\mathbf{r}_{1}-\mathbf{r}_{2}\right|$ and $s=\left|t_{1}-t_{2}\right|$. In addition, $\xi(\mathbf{r}, t)$ is assumed to represent an incompressible flow $[\nabla \xi(\mathbf{r}, t)=0]$ so that [19]

$$
\sum_{j} \frac{\partial R^{i j}(r, s)}{\partial r^{j}}=0 .
$$

Homogeneity and isotropy requirements imply that $R^{i j}(r, 0)$ has to be written in terms of radial functions $f(r)$ and $g(r)$ as

$$
R^{i j}(r, 0)=[f(r)-g(r)] n^{i} n^{j}+g(r) \delta_{i j}
$$

where $n^{i}$ stand for the components of the unit vector in the $\mathbf{r}_{1}-\mathbf{r}_{2}$ direction. Due to the restriction (2.2) applied to $2 \mathrm{D}$ flows, these radial functions are related by

$$
g(r)=f(r)+r \frac{d f(r)}{d r} .
$$

Usually one characterizes an experimental turbulent flow by means of macroscopic quantities defined in terms of the radial correlation

$$
R(r, s) \equiv \frac{1}{2}\left[R^{x x}(r, s)+R^{y y}(r, s)\right] .
$$

Three of these quantities are especially significative. The fist one refers to the intensity of the turbulence $u_{0}^{2}$, measuring the mean energy dissipation rate per unit mass. The characteristic time of the turbulence $t_{0}$ gives the temporal scale of relaxation of the velocity fluctuations. Finally, the characteristic length of the turbulence $l_{0}$ is a convenient measure of the lineal extent through which the local velocities are appreciably correlated. These basic parameters are evaluated in terms of $R(r, s)$ through the definitions

$$
\begin{aligned}
& u_{0}^{2}=R(0,0), \\
& t_{0}=\frac{1}{u_{0}^{2}} \int_{0}^{\infty} d s R(0, s), \\
& l_{0}=\frac{1}{u_{0}^{2}} \int_{0}^{\infty} d r R(r, 0) .
\end{aligned}
$$

At this point, of particular interest for us is the energy spectrum function $E(k)$ representing the density of contributions to $u_{0}^{2}$ on the wave-number axis. In fact, another definition of the turbulent intensity is

$$
u_{0}^{2}=\int_{0}^{\infty} d k E(k) \text {. }
$$

Specific attention to the behavior of $E(k)$ will be reserved for Sec. V.

In order to incorporate all the above properties, we start by defining the velocity field in terms of a scalar field $\eta(\mathbf{r}, t)$, which is assumed to be a homogeneous, isotropic, and stationary Gaussian stochastic process, according to

$$
\begin{aligned}
\boldsymbol{\xi}(\mathbf{r}, t) & =\left(\xi^{x}(\mathbf{r}, t), \xi^{y}(\mathbf{r}, t)\right) \\
& =\left(-\frac{\partial \eta(\mathbf{r}, t)}{\partial y}, \frac{\partial \eta(\mathbf{r}, t)}{\partial x}\right] .
\end{aligned}
$$

By means of Eq. (2.8), the statistics of the incompressible flow $\xi(\mathbf{r}, t)$ is transferred from that of $\eta(\mathbf{r}, t)$. Straightforward algebra shows that the properties (2.3) and (2.4) are recovered after identifying $f(r)$ and $g(r)$ in terms of the stationary correlation of the scalar field $C(r, 0)$ $\equiv\left\langle\eta\left(\mathbf{r}_{1}, t\right) \eta\left(\mathbf{r}_{2}, t\right)\right\rangle$ as

$$
f(r)=-\frac{C^{\prime}(r, 0)}{r}, g(r)=-C^{\prime \prime}(r, 0)
$$

where $C^{n \prime}(r, 0)=d^{n} C(r, 0) / d r^{n}$. By using the standard Fourier transform technique applied to $2 \mathrm{D}$ fields, we obtain a simplified expression for the correlation $R(r, s)$ which reads $[20,21]$

$$
R(r, s)=\frac{1}{4 \pi} \int_{0}^{\infty} d k k^{3} J_{0}(k r) S(k, s)
$$

where $J_{0}(x)$ is the Bessel function of zeroth order and the structure factor $S(k, s)$ stands for the Fourier transform of $C(r, s)$.

What we need to completely specify the stochastic velocity field is the knowledge of $\eta(\mathbf{r}, t)$ and its statistical properties. Our prescription is expressed through a $2 \mathrm{D}$ stochastic Langevin equation, which represents an Ornstein-Uhlenbeck process with a spatial filtering affecting the white-noise term

$$
\tau \frac{\partial \eta(\mathbf{r}, t)}{\partial t}=-\eta(\mathbf{r}, t)+Q\left[\lambda^{2} \nabla^{2}\right] \zeta(\mathbf{r}, t)
$$


where $\zeta(\mathbf{r}, t)$ is a Gaussian white noise of zero mean and correlation

$$
\left\langle\zeta\left(\mathbf{r}_{1}, t_{1}\right) \xi\left(\mathbf{r}_{2}, t_{2}\right)\right\rangle=2 \epsilon \delta\left(\mathbf{r}_{1}-\mathbf{r}_{2}\right) \delta\left(t_{1}-t_{2}\right) .
$$

Equations (2.11) and (2.12) identify the three basic statistical parameters of $\eta(\mathbf{r}, t): \epsilon$ stands for the intensity of the noise whereas $\lambda$ and $\tau$ respectively identify its spatial and temporal correlation lengths. The mathematical operator $Q\left[\lambda^{2} \nabla^{2}\right]$ acting on $\xi(\mathbf{r}, t)$ is such that the Fourier transform of Eq. (2.11) gives a function $Q\left(-\lambda^{2} k^{2}\right)$ multiplying the Fourier transform of the white noise in the form

$$
\tau \frac{\partial \widetilde{\eta}(\mathbf{k}, t)}{\partial t}=-\widetilde{\eta}(\mathbf{k}, t)+Q\left(-\lambda^{2} k^{2}\right) \widetilde{\xi}(\mathbf{k}, t)
$$

where now $\widetilde{\xi}(\mathbf{k}, t)$ is a zero mean $\delta$-functionanticorrelated noise with

$$
\left\langle\widetilde{\xi}\left(\mathbf{k}_{1}, t_{1}\right) \widetilde{\xi}\left(\mathbf{k}_{2}, t_{2}\right)\right\rangle=8 \pi^{2} \epsilon \delta\left(\mathbf{k}_{1}+\mathbf{k}_{2}\right) \delta\left(t_{1}-t_{2}\right) \text {. }
$$

The simplest example for the operator above is $Q\left[\lambda^{2} \nabla^{2}\right]=\lambda^{2} \nabla^{2}$, although more interesting cases will be considered in Sec. V. From Eq. (2.13) we can obtain the desired structure factor $S(k, s)$ whose standard definition is

$$
S(k, s)=\lim _{t \rightarrow \infty} \frac{1}{4 \pi^{2}}\left\langle\widetilde{\eta}^{*}(\mathbf{k}, t) \widetilde{\eta}(\mathbf{k}, t+s)\right\rangle,
$$

which involves the complex conjugate of $\widetilde{\eta}(\mathbf{k}, t)$. After analytical integration of Eq. (2.13) and averaging we obtain

$$
S(k, s)=\frac{\epsilon}{\tau} Q^{2}\left(-\lambda^{2} k^{2}\right) \exp \left(-\frac{s}{\tau}\right) .
$$

It is worth noting that given the spatiotemporal factorization of Eq. (2.16), the correlation $R(r, s)$ will also be factorized. After successive substitution of Eq. (2.16) into Eqs. (2.10), (2.6), and (2.7) we finally obtain all the statistical properties of the turbulent flow. In particular, we directly get for the characteristic time $t_{0}$ and the energy spectrum function the results

$$
\begin{aligned}
& t_{0}=\tau, \\
& E(k)=\frac{\epsilon}{4 \pi \tau} k^{3} Q^{2}\left(-\lambda^{2} k^{2}\right)
\end{aligned}
$$

whereas the correlation length turns out to be proportional to $\lambda$, the specific proportionality being derived for each particular realization of $Q\left[\lambda^{2} \nabla^{2}\right]$.

\section{THE DISCRETE EQUATIONS IN FOURIER SPACE}

The main steps in the generation of the stochastic velocity field are the computer simulation of Eqs. (2.11) and (2.8). Obviously this requires the appropriate discretization of the continuous scheme introduced in Sec. II. We have chosen as a standard grid a two-dimensional square lattice of $N \times N$ mesh points with elementary unit spacing $\Delta_{x}=\Delta_{y}=\Delta$ and shifted periodic boundary conditions [22]. Taking the limit $\Delta \rightarrow 0$ and $N \rightarrow \infty$ one has to recover the results for the continuous model. As anticipat- ed in the Introduction, the simulation of the discrete auxiliary field $\eta_{m n}(t)$ in Fourier space largely facilitates its manipulation. The corresponding discrete Fourier transform is given by

$\widetilde{\eta}_{\mu v}(t)=\Delta^{2} \sum_{m, n} \exp \left[-i(m \mu+n v) \frac{2 \pi}{N}\right] \eta_{m n}(t)$.

From Eq. (3.1), the discrete versions of Eqs. (2.13) and (2.14) transform into

$\tau \frac{d \widetilde{\eta}_{\mu \nu}(t)}{d t}=-\widetilde{\eta}_{\mu v}(t)+Q_{\mu \nu} \widetilde{\xi}_{\mu v}(t)$

$\left\langle\widetilde{\xi}_{\mu \nu}\left(t_{1}\right) \widetilde{\xi}_{\rho \sigma}\left(t_{2}\right)\right\rangle=2 \epsilon(N \Delta)^{2} \delta_{\mu,-\rho} \delta_{v,-\sigma} \delta\left(t_{1}-t_{2}\right)$

where $Q_{\mu \nu}$ is a numerical factor obtained from the Fourier transform of the operator $Q\left[\lambda^{2} \nabla^{2}\right]$ in its discrete version. The great advantage of this procedure is that the Langevin equation (2.11) transforms in a set of decoupled ordinary differential equations which can be exactly integrated in time to give [11]

$$
\begin{aligned}
\widetilde{\eta}_{\mu v}(t)= & \widetilde{\eta}_{\mu \nu}(0) \exp \left[-\frac{t}{\tau}\right] \\
& +\frac{1}{\tau} \int_{0}^{t} d t^{\prime} \exp \left[\frac{t^{\prime}-t}{\tau}\right] Q_{\mu \nu} \xi_{\mu \nu}\left(t^{\prime}\right) .
\end{aligned}
$$

From this expression and Eq. (3.3) one can calculate the discrete structure factor $S_{\mu \nu}(s)$, which explicitly reads

$$
\begin{aligned}
S_{\mu v}(s) & =\lim _{t \rightarrow \infty} \frac{1}{(N \Delta)^{2}}\left\langle\widetilde{\eta}_{\mu \nu}^{*}(t) \widetilde{\eta}_{\mu v}(t+s)\right\rangle \\
& =\frac{\epsilon}{\tau} Q_{\mu \nu}^{2} \exp \left[-\frac{s}{\tau}\right]
\end{aligned}
$$

The appropriate transformation of Eq. (2.8) gives, for the discrete wave-number components of the velocity field,

$$
\begin{aligned}
& \widetilde{\xi}_{\mu v}^{x}(t)=-\frac{i}{\Delta} \sin \left(\frac{2 \pi v}{N}\right) \widetilde{\eta}_{\mu v}(t), \\
& \widetilde{\xi}_{\mu \nu}^{y}(t)=\frac{i}{\Delta} \sin \left(\frac{2 \pi \mu}{N}\right) \widetilde{\eta}_{\mu \nu}(t) .
\end{aligned}
$$

In analogy with Eq. (3.5), the discrete Fourier transform for $R(r, s)$ will be

$$
\begin{aligned}
\widetilde{R}_{\mu \nu}(s)=\lim _{t \rightarrow \infty} \frac{1}{2(N \Delta)^{2}}\left\langle\widetilde{\xi}_{\mu \nu}^{x *}(t) \widetilde{\xi}_{\mu \nu}^{x}(t+s)\right. \\
\\
\left.+\widetilde{\xi}_{\mu \nu}^{y *}(t) \widetilde{\xi}_{\mu \nu}^{y}(t+s)\right\rangle
\end{aligned}
$$

Substituting successively Eqs. (3.6) and (3.5) into Eq. (3.7) and transforming back to real space we finally get 


$$
\begin{aligned}
R_{m n}(s)= & \frac{1}{(N \Delta)^{2}} \sum_{\mu, v} \exp \left[-i(m \mu+n v) \frac{2 \pi}{N}\right) \widetilde{R}_{\mu v}(s) \\
=\frac{\epsilon}{2 \tau N^{2} \Delta^{4}} \sum_{\mu, v} & \exp \left[-i(m \mu+n v) \frac{2 \pi}{N}-\frac{s}{\tau}\right) \\
& \times Q_{\mu v}^{2}\left[\sin ^{2}\left(\frac{2 \pi \mu}{N}\right)+\sin ^{2}\left(\frac{2 \pi v}{N}\right)\right]
\end{aligned}
$$

from which we can identify the three basic parameters of the turbulent flow $u_{0}^{2}, t_{0}$, and $l_{0}$. The result for the intensity of the turbulence reads

$$
\begin{aligned}
u_{0}^{2} & =R_{00}(0) \\
& =\frac{\epsilon}{2 \tau N^{2} \Delta^{4}} \sum_{\mu, v} Q_{\mu v}^{2}\left[\sin ^{2}\left(\frac{2 \pi \mu}{N}\right)+\sin ^{2}\left(\frac{2 \pi v}{N}\right)\right]
\end{aligned}
$$

whereas for $t_{0}$, Eq. (2.17) is recovered. In what refers to the characteristic length of the turbulence, we first perform a least-square fit of $R_{m n}(0)$ to the continuous result $R(r, 0)$ and then, following Eq. (2.6), we analytically integrate the obtained fit from zero to infinity. This procedure will be clarified in Sec. V.

In order to obtain an expression for the energy spectrum function, we will make use of the symmetry of $E(k)$. Taking into account the homogeneity and isotropy properties, Eq. (2.7) can be formally recasted in the form

$$
u_{0}^{2}=\int_{R^{2}} d^{2} \mathbf{k} \frac{E(k)}{2 \pi k}
$$

which when discretized converts into [note that $\left.k_{\mu v}=\left(\mu^{2}+v^{2}\right)^{1 / 2}(2 \pi / N \Delta)\right]$

$$
u_{0}^{2}=\sum_{\mu, v} \frac{1}{N \Delta} \frac{E_{\mu v}}{\left(\mu^{2}+v^{2}\right)^{1 / 2}}
$$

Comparison between Eqs. (3.9) and (3.11) shows us that

$$
\begin{aligned}
E_{\mu \nu}= & \frac{\epsilon}{2 \tau N \Delta^{3}} Q_{\mu \nu}^{2}\left(\mu^{2}+v^{2}\right)^{1 / 2} \\
& \times\left[\sin ^{2}\left(\frac{2 \pi \mu}{N}\right)+\sin ^{2}\left(\frac{2 \pi v}{N}\right)\right] .
\end{aligned}
$$

The next step in the generation of the stochastic velocity field is the specification of the numerical algorithms which correspond to the discrete model of this section. All the results obtained within the simulation scheme should thus be compared with those described above.

\section{SIMULATION ALGORITHMS}

Here we will develop an iterative algorithm appropriate to the discrete version of Eqs. (2.13) and (2.14) which evaluate $\widetilde{\eta}_{\mu v}$ at time $t+\Delta t$ from its known value at time $t$. With these obtained values, we will calculate the statistical quantities characterizing the turbulent flow in terms of averages involving $\widetilde{\eta}_{\mu \nu}(t)$. Integration of Eq. (3.2) between $t$ and $t+\Delta t$ leads to

$$
\begin{aligned}
& \widetilde{\eta}_{\mu \nu}(t+\Delta t)= \widetilde{\eta}_{\mu \nu}(t) \exp \left[-\frac{\Delta t}{\tau}\right] \\
&+\frac{1}{\tau} \int_{t}^{t+\Delta t} d t^{\prime} \exp \left[\frac{t^{\prime}-t-\Delta t}{\tau}\right] \\
& \times Q_{\mu \nu} \widetilde{\xi}_{\mu v}\left(t^{\prime}\right) .
\end{aligned}
$$

If we define a new random variable as [11]

$$
\widetilde{\beta}_{\mu v}(t)=\frac{1}{\tau} \int_{t}^{t+\Delta t} d t^{\prime} \exp \left(\frac{t^{\prime}-t-\Delta t}{\tau}\right) Q_{\mu \nu} \xi_{\mu v}\left(t^{\prime}\right),
$$

this random quantity is Gaussian and has zero mean. Its variance at equal times can be evaluated from Eq. (3.3) to give

$$
\begin{aligned}
& \left\langle\widetilde{\beta}_{\mu v}(t) \widetilde{\beta}_{\rho \sigma}(t)\right\rangle \\
& =\frac{\epsilon(N \Delta)^{2}}{\tau} Q_{\mu \nu}^{2}\left[1-\exp \left[-\frac{2 \Delta t}{\tau}\right]\right] \delta_{\mu,-\rho} \delta_{v,-\sigma} .
\end{aligned}
$$

From Eq. (4.3) and assuming that $Q_{\mu, v}=Q_{-\mu,-v}, \widetilde{\beta}_{\mu v}(t)$ can then be expressed as

$$
\widetilde{\beta}_{\mu \nu}(t)=(N \Delta) Q_{\mu \nu}\left\{\frac{\epsilon}{\tau}\left[1-\exp \left[-\frac{2 \Delta t}{\tau}\right]\right]\right\}^{1 / 2} \widetilde{\alpha}_{\mu \nu}(t)
$$

where $\widetilde{\alpha}_{\mu v}(t)$ are Gaussian anticorrelated random numbers such that

$$
\left\langle\widetilde{\alpha}_{\mu v}(t) \widetilde{\alpha}_{\rho \sigma}(t)\right\rangle=\delta_{\mu,-\rho} \delta_{v,-\sigma}
$$

and whose simulation scheme has been learned from the existing literature [11]. As a result, the desired numerical algorithm is then

$$
\widetilde{\eta}_{\mu \nu}(t+\Delta t)=\widetilde{\eta}_{\mu v}(t) \exp \left[-\frac{\Delta t}{\tau}\right]+\widetilde{\beta}_{\mu \nu}(t) .
$$

The initial condition $\widetilde{\eta}_{\mu \nu}(0)$ is chosen to correspond to that of the steady state (here understood in a statistical sense). In this way we are sure to be in an isotropic and homogeneous velocity steady state from the beginning of the simulation. Looking at Eq. (3.5), this is accomplished by taking

$$
\widetilde{\eta}_{\mu v}(0)=(N \Delta) Q_{\mu v}\left(\frac{\epsilon}{\tau}\right)^{1 / 2} \widetilde{\alpha}_{\mu \nu}(0) .
$$

The numerical running of these expressions (and those appearing below) is advantageously vectorized by transforming the 2D lattice to a $1 \mathrm{D}$ array using a single index $\kappa=(\mu-1) N+\nu$. The same procedure is applied to real space.

Once $\widetilde{\eta}_{\mu v}(t)$ is obtained we can either evaluate the stochastic flow or its statistical properties. In the first case we transform $\widetilde{\eta}_{\mu \nu}(t)$ back to real space as

$\eta_{m n}(t)=\frac{1}{(N \Delta)^{2}} \sum_{\mu, v} \exp \left[-i(m \mu+n v) \frac{2 \pi}{N}\right) \widetilde{\eta}_{\mu \nu}(t)$ 
and from it we obtain the components of the stochastic turbulence using discrete centered forms appropriate to Eq. (2.8)

$$
\begin{aligned}
& \xi_{m, n}^{x}(t)=\frac{1}{2 \Delta}\left[\eta_{m, n-1}(t)-\eta_{m, n+1}(t)\right], \\
& \xi_{m, n}^{y}(t)=\frac{1}{2 \Delta}\left[\eta_{m+1, n}(t)-\eta_{m-1, n}(t)\right],
\end{aligned}
$$

where shifted periodic boundary conditions are explicitly used. In what refers to the statistical properties, the quantities of interest are given by

$$
\begin{aligned}
R_{m n}(s)=\frac{1}{2 N^{4} \Delta^{6}} \sum_{\mu, v} & \exp \left[-i(m \mu+n v) \frac{2 \pi}{N}-\frac{s}{\tau}\right] \\
\times & {\left[\sin ^{2}\left(\frac{2 \pi \mu}{N}\right)+\sin ^{2}\left(\frac{2 \pi v}{N}\right)\right] } \\
& \times\left\langle\widetilde{\eta}_{\mu \nu}^{*}(t) \widetilde{\eta}_{\mu \nu}(t+s)\right\rangle
\end{aligned}
$$

and

$$
\begin{aligned}
E_{\mu \nu}= & \frac{1}{2 N^{3} \Delta^{5}}\left(\mu^{2}+v^{2}\right)^{1 / 2}\left[\sin ^{2}\left(\frac{2 \pi \mu}{N}\right]+\sin ^{2}\left[\frac{2 \pi v}{N}\right]\right] \\
& \times\left\langle\widetilde{\eta}_{\mu \nu}^{*}(t) \widetilde{\eta}_{\mu v}(t)\right\rangle
\end{aligned}
$$

where averages are taken over realizations of the stochastic flow. The results obtained from Eqs. (4.10) and (4.11) should be compared with Eqs. (3.8) and (3.12).

\section{EXAMPLES}

Up to now we have introduced a general scheme suitable to generate incompressible, homogeneous, isotropic, and stationary 2D velocity fields. What is discussed here is the application of this general scheme to appropriate spectral realizations of particular turbulent flows. The kind of spectra we are interested in belong to the class which could be classified as being well behaved. This is understood here in the sense that they reproduce to a good extent the main features of experimental energy spectra. Two examples have been chosen for $E(k)$. The first and simplest one is that proposed by Kraichnan [3], which describes a widely distributed band of excitations with a peak centered at a well-defined wave number $k_{0}$

$$
E(k) \propto k^{3} \exp \left(-\frac{k^{2}}{k_{0}^{2}}\right) .
$$

The second example is intended to reproduce the universal Kolmogorov's inertial subrange, characterized by the well-known " $-\frac{5}{3}$ " law [23]. To this end we select a Kármán-Obukhov spectra, whose general behavior is $[16,17]$

$$
E(k) \propto k^{n}\left(1+\frac{k^{2}}{k_{0}^{2}}\right)^{-(5+3 n) / 6},
$$

which for $k$ large enough reproduces the desired inertial subrange. For convenience we have taken $n=3$. The two selected spectra (5.1) and (5.2) will be presented separately.

\section{Case I: Kraichnan's spectrum}

To obtain Eq. (5.1), the choice for the operator $Q$ appearing in Eq. (2.11) is

$$
Q\left[\lambda^{2} \nabla^{2}\right]=\exp \left(\frac{1}{2} \lambda^{2} \nabla^{2}\right)
$$

which gives rise to

$$
Q\left(-\lambda^{2} k^{2}\right)=\exp \left(-\frac{1}{2} \lambda^{2} k^{2}\right) \text {. }
$$

When substituting Eq. (5.4) into the general expressions of Sec. II we get

$$
\begin{aligned}
& R(r, s)=\frac{\epsilon}{8 \pi \tau \lambda^{4}}\left[1-\frac{r^{2}}{4 \lambda^{2}}\right) \exp \left[-\frac{r^{2}}{4 \lambda^{2}}-\frac{s}{\tau}\right], \\
& E(k)=\frac{\epsilon}{4 \pi \tau} k^{3} \exp \left(-\lambda^{2} k^{2}\right)
\end{aligned}
$$

with the following results for the three basic parameters

$$
u_{0}^{2}=\frac{\epsilon}{8 \pi \tau \lambda^{4}}, \quad t_{0}=\tau, \quad l_{0}=\frac{\pi^{1 / 2}}{2} \lambda .
$$

In what refers to the energy spectrum, the characteristic wave number reads

$$
k_{0}=\left(\frac{3}{2}\right)^{1 / 2} \lambda^{-1} \text {. }
$$

When discretizing, the fundamental expression to be substituted into Eqs. (3.8) and (3.12) comes from the discrete version of Eq. (5.4), which translates into

$Q_{\mu \nu}=\exp \left\{-\frac{\lambda^{2}}{\Delta^{2}}\left[2-\cos \left(\frac{2 \pi \mu}{N}\right)-\cos \left(\frac{2 \pi v}{N}\right)\right]\right\}$.

In the following, we will present continuous, discrete, and simulation results corresponding to Kraichnan's spectrum. This first interest refers to the comparison between these three approaches in order to check the goodness of our procedure. Before going to the statistical properties, we briefly show in Figs. 1 and 2 simulation results obtained for individual realizations of the stochastic velocity field $\xi(\mathbf{r}, t)$. In Fig. 1 , the entire square lattice displays the presence of flow lines and eddies of different size and shape subjected to the prescribed shifted periodic boundary conditions. The basic difference between Figs. 1(a) and 1(b) is the mean eddy size. Actually, this size is directly related to $l_{0}$, which is proportional to $\lambda$ [see Eq. (5.7)]. Accordingly, and for the sake of comparison, we have plotted $\lambda \times \lambda$ squares over typical eddies.

Shown in Fig. 2 are two snapshots of a particular realization of the stochastic turbulence taken at different times. They correspond to a piece of the square lattice and give us an idea on how fluid moves subject to the incompressibility condition.

Referring to the statistical flow properties obtained through averages of individual realizations, the first quantity of interest is the radial correlation $R(r, s)$. As the discretization only concerns spatial degrees of freedom, we will not present any result which implies averages at different times. Depicted in Fig. 3 is the behavior of $R(r, 0)$ vs $r$ for different values of $\lambda$, evaluated in the 
continuous and simulation approaches. As shown, the agreement between simulation and continuous results is rewarding, especially for appropriate values (see below) of $\lambda / \Delta$. We have not plotted the discrete results since, for high enough number of realizations, simulation collapses on the discrete scheme. Thus, hereafter, we will only compare the continuous and discrete models. In this respect, and given the used boundary conditions, we can anticipate that the goodness of the discretization will essentially depend on the quotient $\lambda / \Delta$ relative to the number of mesh points $N$. This question is explicitly exemplified by referring to the value $l_{0}$ (discrete) $/ l_{0}$ (continuous).

Before going further, it is necessary to clarify how
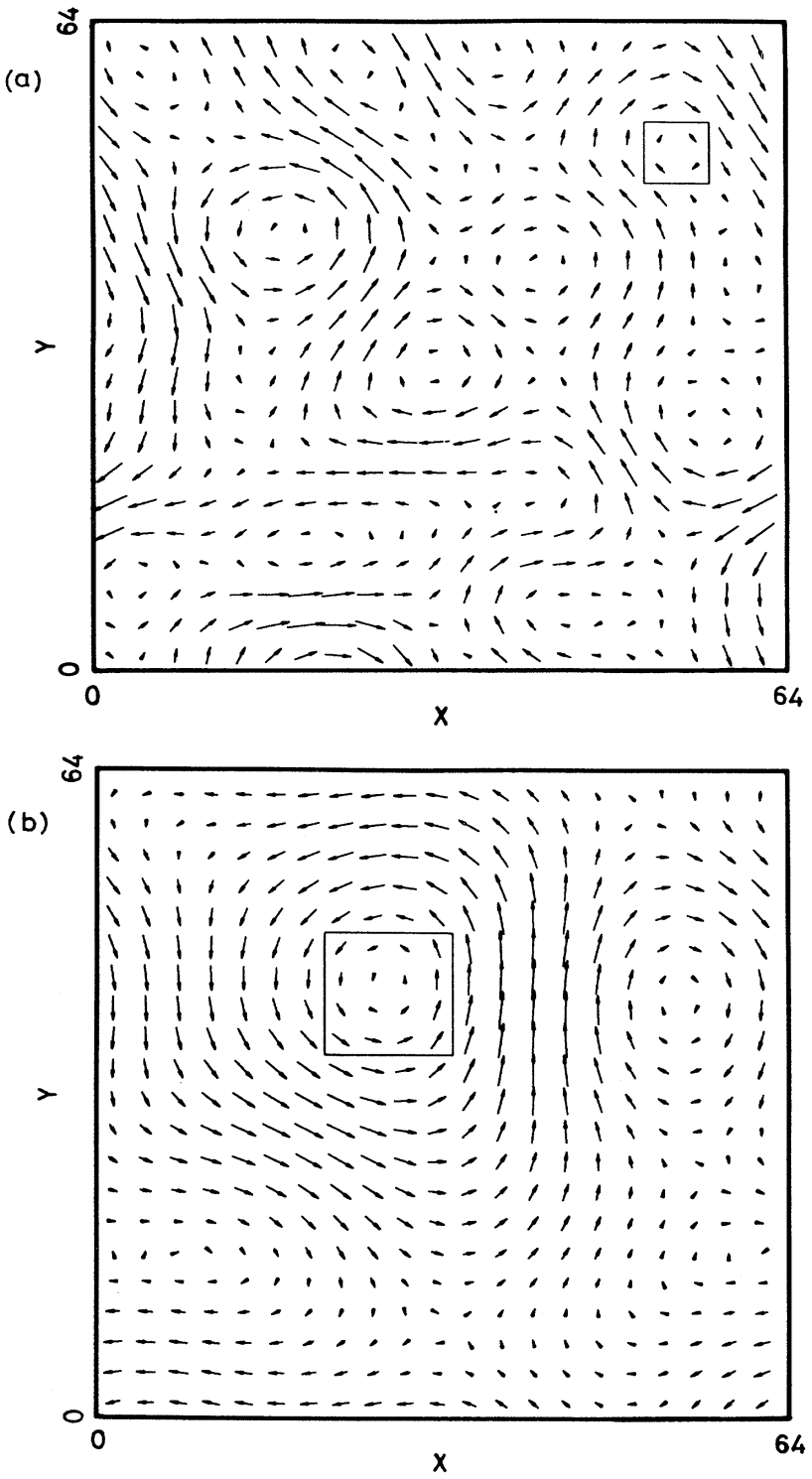

FIG. 1. Simulation patterns for particular realizations of the stochastic velocity field $\xi(\mathbf{r}, t)$ corresponding to case I with $\epsilon=10.0, \tau=1.0, \Delta=1.0, \Delta t=0.1$, and $64 \times 64$ mesh points. (a) $\lambda=6.0$ and (b) $\lambda=12.0$. The inner squares' area is $\lambda \times \lambda$. $l_{0}$ (discrete) is obtained. As anticipated in Sec. III, we perform a least-square fit of $R_{m n}(0)$ in the form

$$
R_{m n}(0)=\frac{\epsilon}{8 \pi \tau \lambda_{\text {fit }}^{4}}\left(1-\frac{r_{m n}^{2}}{4 \lambda_{\text {fit }}^{2}}\right) \exp \left(-\frac{r_{m n}^{2}}{4 \lambda_{\text {fit }}^{2}}\right)
$$

where the parameter to be fitted is $\lambda_{\text {fit }}$. Note that $r_{m n}=\left(m^{2}+n^{2}\right)^{1 / 2} \Delta$. In order to avoid finite size effects, we have extended the fit over the interval $0 \leq r_{m n} \leq N \Delta / 4$. Once $\lambda_{\text {fit }}$ is obtained, we simply have

$$
\frac{l_{0}(\text { discrete })}{l_{0}(\text { continuous })}=\frac{\lambda_{\text {fit }}}{\lambda} \text {. }
$$
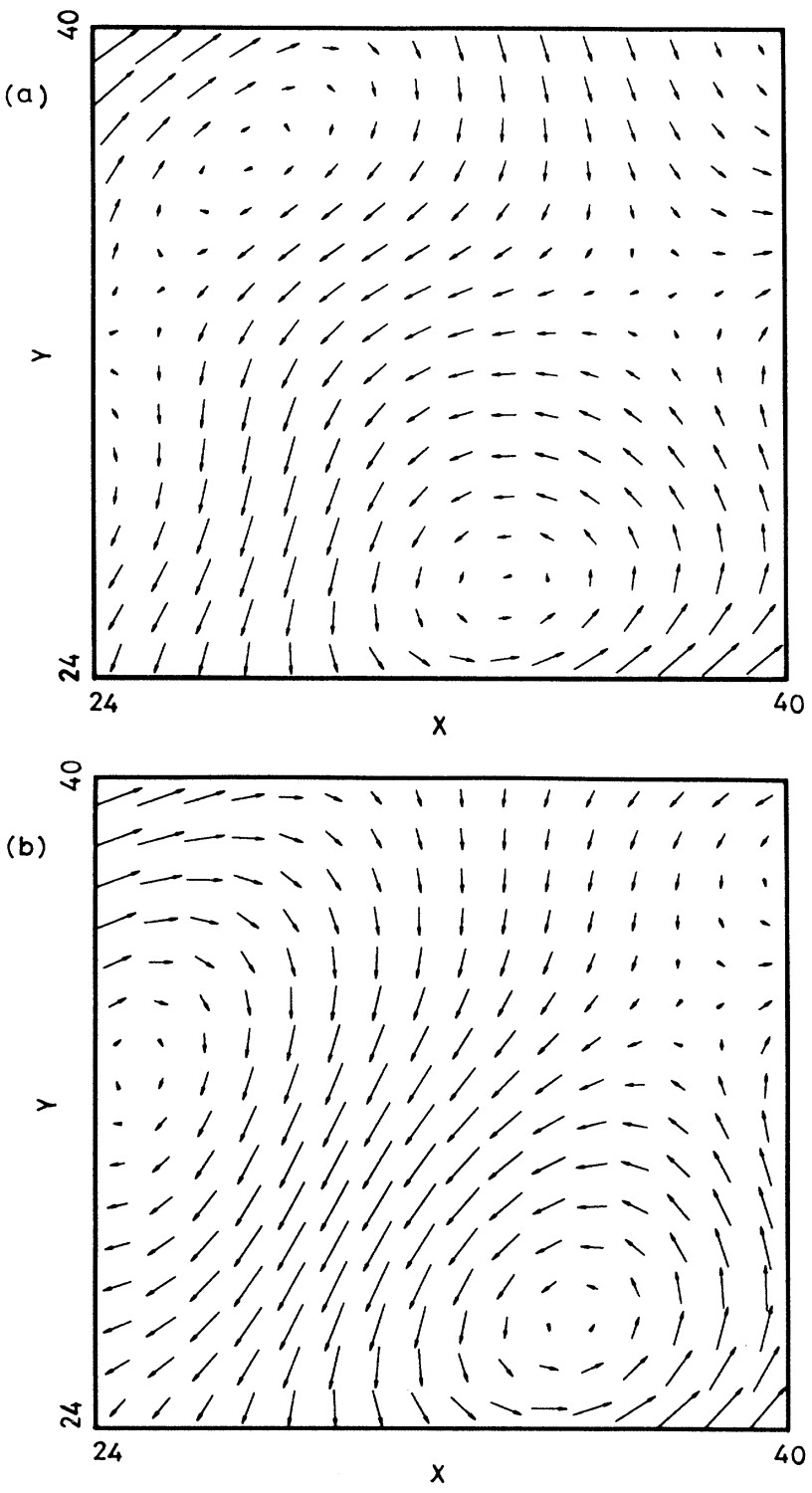

FIG. 2. Snapshots of simulation patterns similar to those of Fig. 1 taken at different times. Parameter values are $\epsilon=10.0$, $\tau=1.0, \lambda=4.0, \Delta=1.0, \Delta t=0.1$, and $64 \times 64$ mesh points. (a) $t=0.2$ and (b) $t=0.4$. 


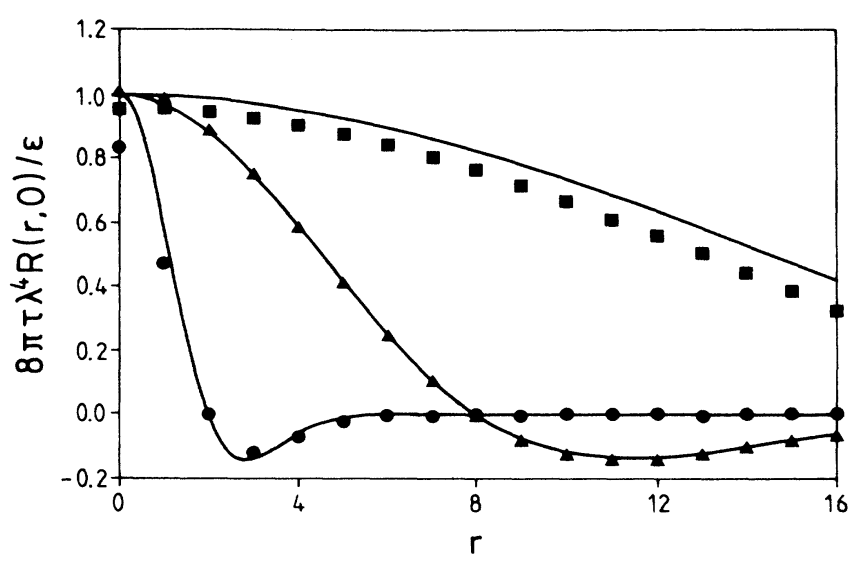

FIG. 3. Normalized radial correlation function $R(r, 0)$ vs $r$ corresponding to case I for different values of $\lambda$. Solid lines correspond to Eq. (5.5) and symbols are simulation results obtained with $\epsilon=10.0, \tau=1.0, \Delta=1.0, \Delta t=0.1$, and $64 \times 64 \mathrm{mesh}$ points. (O) $\lambda=1.0,(\Delta) \lambda=4.0$, and (ロ) $\lambda=13.0$. Averages are taken over 1000 realizations.

Summarized in Fig. 4 are the results obtained for Eq. (5.11) vs $\lambda / \Delta$ for different values of $N$, together with the values obtained for the three simulation cases of Fig. 3. As expected, they appear to be sensitive only to the value $\lambda / \Delta$. When $\lambda / \Delta \approx 1$, the discrete model is not able to properly reproduce features involving distances or order $\Delta$. On the other hand, finite-size effects begin to play a role for high enough values of $\lambda / \Delta$ compared to $N$. In between these two regions, discretization appears to be satisfactory provided that

$$
1<\frac{\lambda}{\Delta} \leq \frac{1}{4} N \text {. }
$$

When one refers to the value $u_{0}^{2}$ (discrete) $/ u_{0}^{2}$ (continuous),

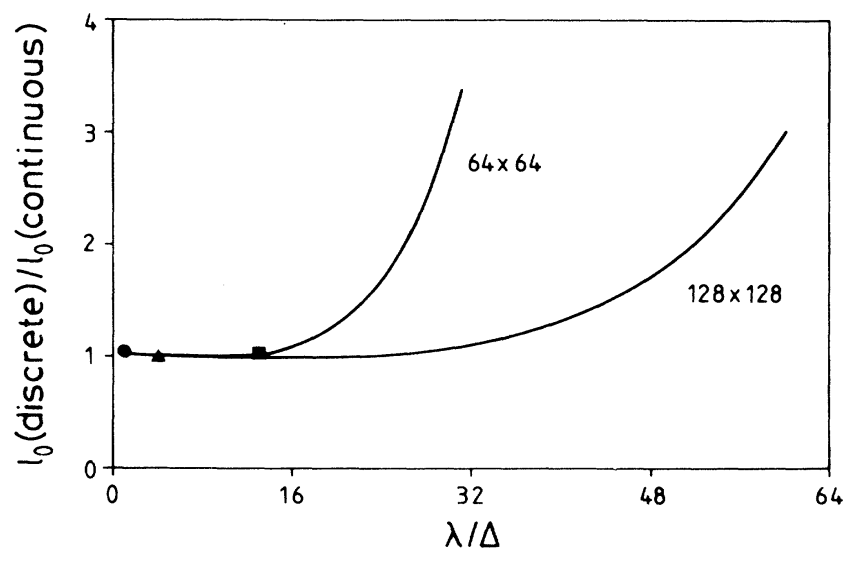

FIG. 4. Relative length $l_{0}$ (discrete) $/ l_{0}$ (continuous) vs $\lambda / \Delta$ corresponding to case $I$ for different number of mesh points. The parameter values are $\epsilon=10.0, \tau=1.0, \Delta=1.0$, and $\Delta t=0.1$. Symbols are the simulation results obtained from Fig. 3.

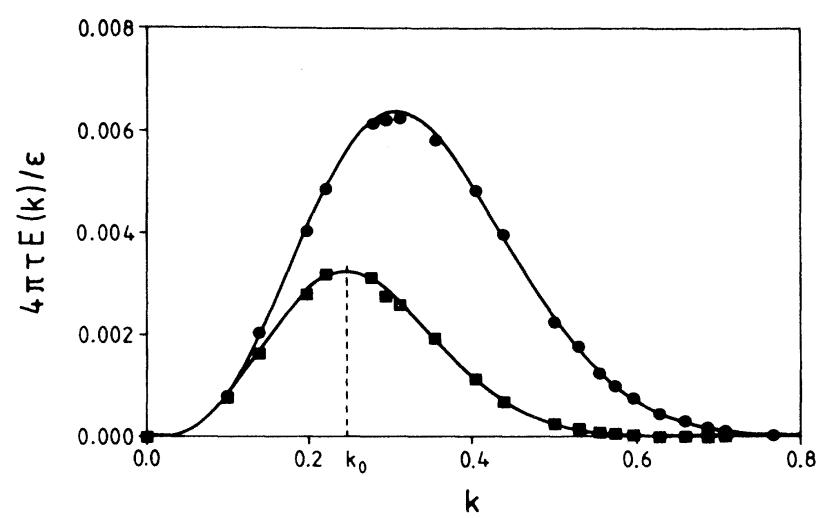

FIG. 5. Normalized energy spectrum function $E(k)$ vs $k$ corresponding to case $I$ for different values of $\lambda$. Solid lines correspond to Eq. (5.6) and symbols are simulation results obtained with $\epsilon=10.0, \tau=1.0, \Delta=1.0, \Delta t=0.1$, and $64 \times 64$ mesh points. (O) $\lambda=4.0$ and $(\boldsymbol{\square}) \lambda=5.0$. Averages are taken over 10000 realizations.

the same rule is obtained, each intensity being evaluated respectively from Eqs. (3.9) and (2.6).

Finally, the characteristic shape of Kairchnan's spectrum is plotted in Fig. 5 for both the continuous and simulation approaches. Also shown is the peak centered at the wave number $k_{0}$ for one of the curves.

\section{Case II: Kármán-Obukhov's spectrum}

For this spectrum we start by taking

$$
Q\left[\lambda^{2} \nabla^{2}\right]=\left(1-\lambda^{2} \nabla^{2}\right)^{-7 / 6}
$$

from which we now obtain

$$
Q\left(-\lambda^{2} k^{2}\right)=\left(1+\lambda^{2} k^{2}\right)^{-7 / 6}
$$

and we derive, for $R(r, s)$ and $E(k)$, the results [20,21]

$$
\begin{aligned}
R(r, s) & =\frac{9 \epsilon}{2^{13 / 3} \Gamma\left(\frac{1}{3}\right) \pi \tau \lambda^{4}}\left[\frac{4}{3} \rho^{1 / 3} K_{1 / 3}(\rho)-\frac{1}{2} \rho^{4 / 3} K_{4 / 3}(\rho)\right] \\
& \times \exp \left[-\frac{s}{\tau}\right] \\
E(k) & =\frac{\epsilon}{4 \pi \tau} k^{3}\left(1+\lambda^{2} k^{2}\right)^{-7 / 3}
\end{aligned}
$$

where $K_{v}(x)$ is the modified Bessel function of fractional order and $\rho \equiv r / \lambda$. The corresponding results for the macroscopic parameters are, in this case,

$$
u_{0}^{2}=\frac{9 \epsilon}{32 \pi \tau \lambda^{4}}, \quad t_{0}=\tau, \quad l_{0}=\frac{\Gamma\left(\frac{1}{2}\right) \Gamma\left(\frac{5}{6}\right)}{2 \Gamma\left(\frac{1}{3}\right)} \lambda .
$$

Analogously to case I, we can define a characteristic wave number $k_{0}$ as the maximum of $E(k)$. For Kármán-Obukhov's spectrum, one obtains the value

$$
k_{0}=\left(\frac{9}{5}\right)^{1 / 2} \lambda^{-1} \text {. }
$$

The discrete version of Eq. (5.14) to be used in the discretized scheme reads 


$$
\begin{gathered}
Q_{\mu v}=\left\{1+\frac{2 \lambda^{2}}{\Delta^{2}}\left[2-\cos \left[\frac{2 \pi \mu}{N}\right]\right.\right. \\
\left.\left.-\cos \left[\frac{2 \pi v}{N}\right)\right]\right\}^{-7 / 6} .
\end{gathered}
$$

The most significative feature of this second example is the presence of long tails in the energy spectra, as it is shown in Fig. 6(a), where $E(k)$ is plotted against $k$ for different values of $\lambda$. Consequently, in order to obtain reliable simulation results it will be necessary to consider a smaller unit spacing $\Delta$ and a corresponding higher $N$. Depicted in Fig. 6(b) is the log-log plot of Fig. 6(a), showing a linear behavior at high wave numbers. For the sake of comparison, an arbitrary straight line of slope $-\frac{5}{3}$ has been included.

When going to real space, the presence of a broad inertial subrange translates into a radial correlation $R(r, 0)$ which has a peak at the origin, as it is illustrated in Fig. 7. This behavior is clearly opposite to case I, where a short-ranged spectrum (see Fig. 5) converts into a smooth radial correlation near the origin (see Fig. 3). Thus
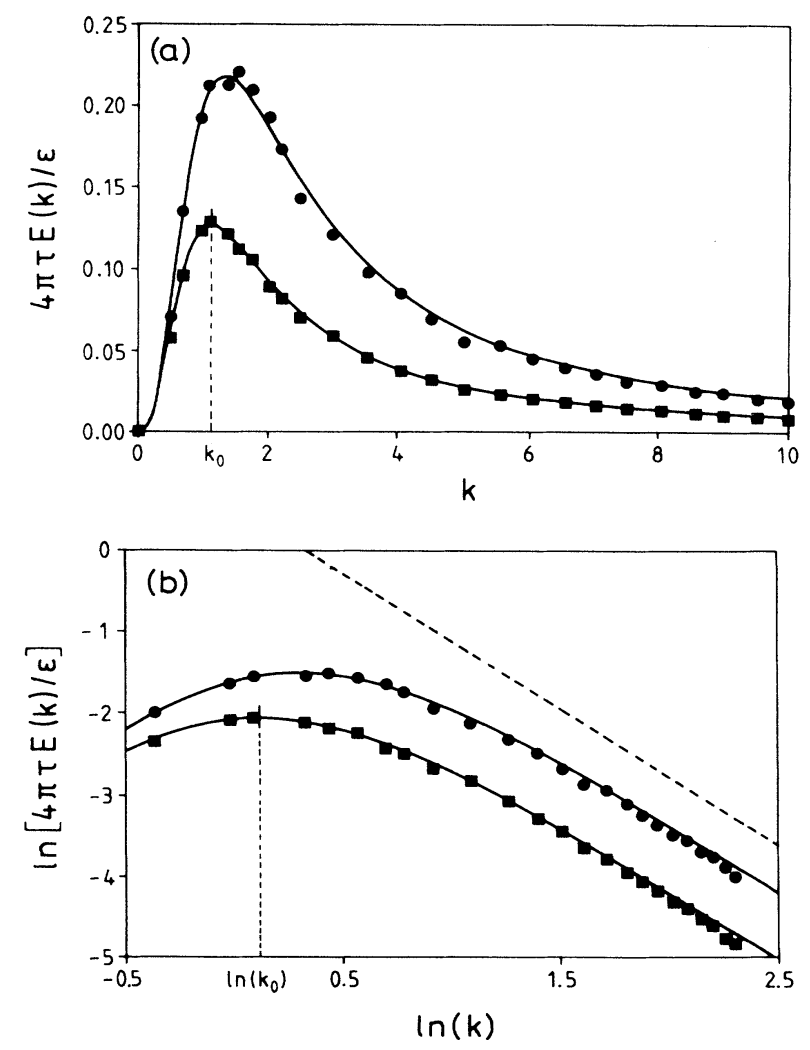

FIG. 6. (a) Normalized energy spectrum function $E(k)$ vs $k$ corresponding to case II for different values of $\lambda$. Solid lines correspond to Eq. (5.16) and symbols are simulation results obtained with $\epsilon=10.0, \tau=1.0, \Delta=0.1, \Delta t=0.1$, and $128 \times 128$ mesh points. (O) $\lambda=1.0$ and (⿶) $\lambda=1.2$. Averages are taken over 10000 realizations. (b) Log-log plot corresponding to (a) with an additional arbitrary straight line of slope $-\frac{5}{3}$.

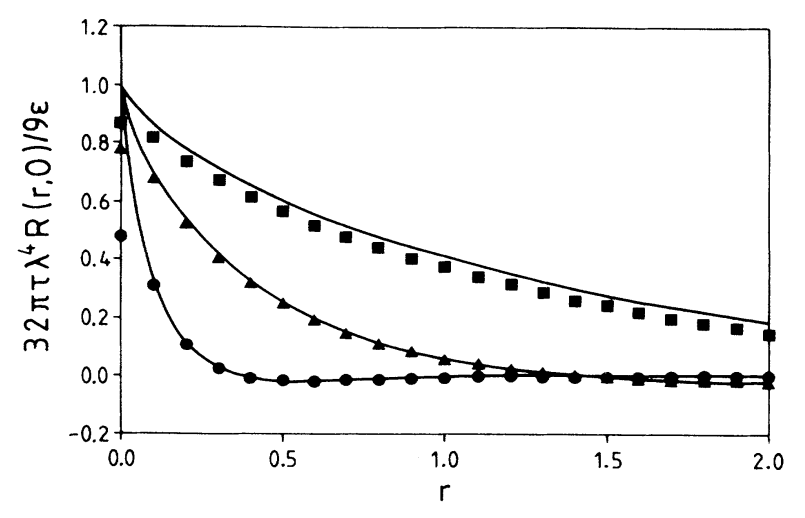

FIG. 7. Normalized radial correlation function $R(r, 0)$ vs $r$ corresponding to case II for different values of $\lambda$. Solid lines correspond to Eq. (5.15) and symbols are simulation results obtained with $\epsilon=10.0, \tau=1.0, \Delta=0.1, \Delta t=0.1$, and $128 \times 128$ mesh points. (O) $\lambda=0.2$, ( $\boldsymbol{\Delta}) \lambda=0.8$, and (ם) $\lambda=2.6$. Averages are taken over 1000 realizations.

Kármán-Obukhov's spectrum shows a richer variety of turbulent structures at short distances which the discretization is unable to correctly reproduce except under the limit of vanishing $\Delta$. That is why simulation results differ significantly from their continuous counterpart at the origin (see Fig. 7) for all values of $\lambda$. Apart from that, the comments above relative to the agreement between discrete and simulation results would apply. The examination of a particular velocity pattern, such as that shown in Fig. 8, points on that $R(r, 0)$ is weaker at short distances (neighboring arrows are less ordered than in Figs. 1 and 2). Besides, eddies are more diffuse accord-

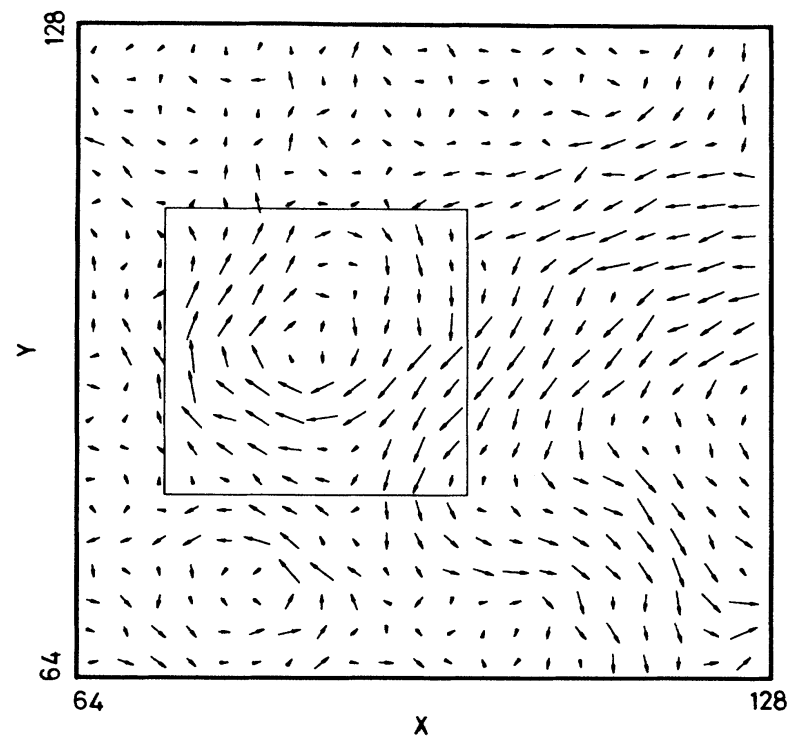

FIG. 8. Partial view of the simulation pattern for a particular realization of the stochastic velocity field $\xi(\mathbf{r}, t)$ corresponding to case II with $\epsilon=10.0, \tau=1.0, \lambda=2.8, \Delta=0.1, \Delta t=0.1$, and $128 \times 128$ mesh points. The inner square's area is $\lambda \times \lambda$. 


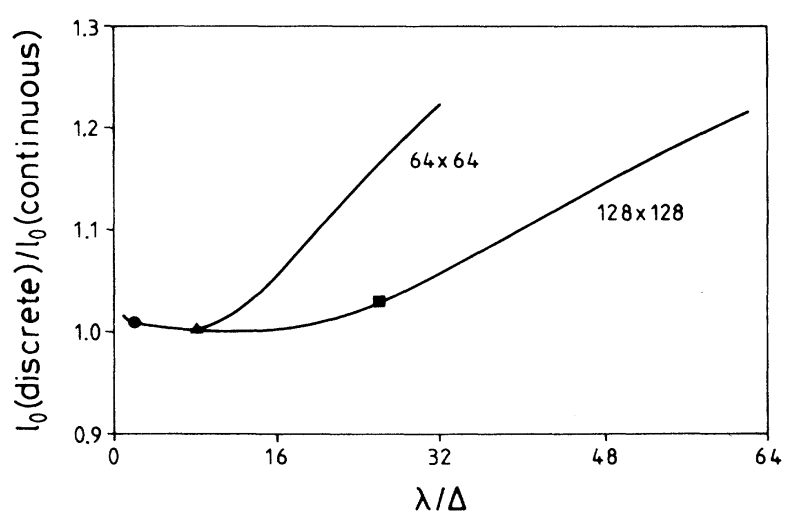

FIG. 9. Relative length $l_{0}$ (discrete) $/ l_{0}$ (continuous) vs $\lambda / \Delta$ corresponding to case II for different number of mesh points. The parameter values are $\epsilon=10.0, \tau=1.0, \Delta=0.1$, and $\Delta t=0.1$. Symbols are the simulation results obtained from Fig. 7.

ing to the fact that the spectrum around $k_{0}$ is much more broader than in case $I$.

Finally, as for case I, we analyze the goodness of the procedure by looking at the value $l_{0}$ (discrete)/ $l_{0}$ (continuous) and, analogously, we will proceed through a least-square fit of $R_{m n}(0)$ equivalent to that of Eq. (5.10). The main difference is that given the discrepancies for the radial correlation at the origin (see Fig. 7), the fit is extended over the interval $\Delta \leq r_{m n} \leq N \Delta / 4$. Once again, the results only depend on the value of $\lambda / \Delta$ relative to $N$. They are summarized in Fig. 9. As in the former case, we can differentiate three distinct regions, the reliable one being bounded by

$$
1<\frac{\lambda}{\Delta} \leq \frac{1}{6} N
$$

which is certainly shorter than that of Eq. (5.12).

\section{CONCLUDING REMARKS}

What we have presented here is a general model aimed at obtaining homogeneous, isotropic, and stationary 2D stochastic velocity fields which reproduce the most significative statistical features of fully developed turbulence. Accordingly, we have mainly focused on the evaluation of the energy spectra and the spatiotemporal correlation functions. Based on the use of stochastic differential equations containing space-time correlated noise, we have derived both analytical expressions in the continuous and discrete space and their corresponding simulation algorithms. By means of simulating the dynamics of the auxiliary scalar field in Fourier space, it is shown that the discretization process is exact in time and consequently it only concerns the transformation from continuous to discrete space.

To illustrate the application of the general scheme, results for two relevant spectra, respectively proposed by Kraichnan and by Kármán and Obhukov, are given. In both cases, the goodness of the computed quantities essentially depends, for a confident enough statistics, on the quotient $\lambda / \Delta$ relative to the number of mesh points $N$.

Finally, it is worth noting that most of the simulation models reviewed in the Introduction have been applied in the context of turbulent diffusion of passive scalars. We hope that with the aid of the simple simulation procedure here developed, a wider class of phenomena could be studied apart from those mentioned in the Introduction. This would be the case, for instance, when dealing with physicochemical systems incorporating reaction, diffusion, and convection of chemical species.

\section{ACKNOWLEDGMENTS}

All the calculations are performed on the IBM-3090 computer at CESCA (Centre de Supercomputació de Catalunya). We acknowledge financial support from Dirección General de Investigación Científica y Técnica (Spain) under Projects Nos. PB90-0030 and PB90-0455.
[1] J. H. Ferziger, J. Comput. Phys. 69, 1 (1987).

[2] R. S. Rogallo and P. Moin, Annu. Rev. Fluid Mech. 16, 99 (1984).

[3] R. H. Kraichnan, Phys. Fluids 13, 22 (1970).

[4] R. H. Kraichnan, J. Fluid Mech. 77, 753 (1976).

[5] I. T. Drummond, S. Duane, and R. R. Horgan, J. Fluid Mech. 138, 75 (1984).

[6] J. C. H. Fung, J. C. R. Hunt, N. A. Malik, and R. J. Perkins, J. Fluid Mech. 236, 281 (1992).

[7] M. Avellaneda and S. Torcuato, Phys. Fluids A 3, 1880 (1991).

[8] J. P. Lynov, A. H. Nielsen, H. L. Pécseli, and J. J. Rasmussen, J. Fluid Mech. 224, 485 (1991).

[9] D. C. Haworth and S. B. Pope, Phys. Fluids 29, 387 (1986).

[10] H. Kaplan and N. Dinar, J. Comput. Phys. 79, 317 (1988).

[11] J. García-Ojalvo, J. M. Sancho, and L. Ramírez-Piscina, Phys. Rev. A 46, 4670 (1992).

[12] A. Careta, J. M. Sancho, and F. Sagués (unpublished).

[13] J. D. Gunton, M. San Miguel, and P. S. Sahni, in Phase Transitions and Critical Phenomena, edited by C. Domb and J. L. Lebowitz (Academic, New York, 1983).

[14] M. Kardar, G. Parisi, and Y. C. Zhang, Phys. Rev. Lett. 56, 889 (1986), E. Medina, T. Hwa, M. Kardar, and Y. C. Zhang, Phys. Rev. A 39, 3053 (1989).

[15] A. S. Mikhailov, Phys. Rep. 184, 307 (1989).

[16] T. von Kármán, Proc. Natl. Acad. Sci. U.S.A. 34, 530 (1948).

[17] A. M. Obukhov, C. R. Acad. Sci. USSR 32, 19 (1941).

[18] A. S. Monin and A. M. Yaglom, Statistical Fluid Mechanics of Turbulence (MIT Press, Cambridge, MA, 1975).

[19] G. K. Batchelor, The Theory of Homogeneous Turbulence (Cambridge University Press, Cambridge, 1970).

[20] I. S. Gradshteyn and I. M. Ryzhik, Table of Integrals, Series and Products, edited by A. Jeffrey (Academic, New York, 1965).

[21] Handbook of Mathematical Functions, edited by $\mathbf{M}$. Abramowitz and I. A. Stegun (Dover, New York, 1972).

[22] K. Binder and D. W. Heermann, Monte Carlo Simulation in Statistical Physics (Springer-Verlag, Berlin, 1988).

[23] A. N. Kolmogorov, C. R. Acad. Sci. USSR 30, 301 (1941). 